\title{
Departiment of Economics
} and Management \section{Discussion} Paper

\section{LCL 2021-02}

\section{Logistics}

\section{Carrier collaboration with endogenous networks: Or, the limits of what carrier collaboration can achieve under antitrust immunity}

available online : https://wwwfr.uni.lu/recherche/fdef/dem/publications/discussion_papers

Achim I. Czerny, The Hong Kong Polytechnic University, HK Peter-J. Jost, WHU - Otto Beisheim School of Management, GE Hao Lang, The Hong Kong Polytechnic University, HK

Benny Mantin, LCL,University of Luxembourg, LU

March, 2021

For editorial correspondence, please contact: dem@uni.lu

University of Luxembourg

Faculty of Law, Economics and Finance

6, Rue Richard Coudenhove-Kalergi

L-1359 Luxembourg

The opinions and results mentioned in this paper do not reflect the position of the Institution 


\title{
Carrier collaboration with endogenous networks: Or, the limits of what carrier collaboration can achieve under antitrust immunity ${ }^{1}$
}

\author{
Achim I. Czerny ${ }^{2}$, Peter-J. Jost ${ }^{3}$, Hao Lang ${ }^{4}$ and Benny Mantin ${ }^{5}$
}

June 25, 2020

\footnotetext{
${ }^{1}$ We thank the participants of the International Forum on Shipping, Ports and Airports 2019 and the GARS Junior Workshop 2020 and especially Volodymyr Bilotkach and Alberto Gaggero for their comments and suggestions.

${ }^{2}$ Department of Logistics and Maritime Studies, The Hong Kong Polytechnic University, Hong Kong, achim.czerny@polyu.edu.hk.

${ }^{3} \mathrm{WHU}$ - Otto Beisheim School of Management, peter-j.jost@whu.edu.

${ }^{4}$ Department of Logistics and Maritime Studies, The Hong Kong Polytechnic University, hao.lang@connect.polyu.hk.

${ }^{5}$ University of Luxembourg, benny.mantin@uni.lu.
} 


\begin{abstract}
Airlines provide complex route networks that are to large extents complementary. Therefore, some passengers need to change aircraft and airlines to fly from their origin to their final destination. The present study captures pricing problems in terms of double marginalization but goes one step further by incorporating scheduling problems in the form of route developments and/or frequency choices. The model involves a two-stage game with two carriers who choose their complementary networks in the first stage and prices in the second stage. Each carrier's network involves one or two routes that are distributed geographically or distributed in time. If both carriers maintain two routes, then transfer passengers can choose between two alternative connections which they consider as imperfect substitutes. There are only transfer passengers, and maintaining a route is costly. The analysis reveals that carrier collaboration and antitrust immunity can eliminate double marginalization and create additional incentives to maintain two connections rather than one connection depending on the heterogeneity of connections. Our results indicate that the scope for the improvement of carrier networks via antritrust immunity can be rather limited relative to the social desirability of more complete carrier networks. A possible policy lesson is that airlines should be granted antitrust immunity conditional on network expansion.
\end{abstract}

Keywords: Carriers; networks; frequencies; double marginalization; antitrust immunity; service obligations 


\section{Introduction}

Airlines provide complex route networks that are to large extents complementary. One reason for the complementarity is related to bilateral service agreements, which restrict the carriers' access to the national air space of foreign countries and their airports (for example, Czerny and Lang, 2019). The complementarity implies that some passengers need to interline in the sense that they must change aircraft and carrier to fly from their origin to their final destination. However, such complementarity raises the issue of a "chain of non-integrated concerns" as was highlighted by Spengler (1950). More specifically, uncoordinated airline pricing leads to excessive prices via double marginalization which can be avoided by granting antitrust immunity to airlines allowing for coordinated pricing decisions (for example, Brueckner and Whalen, 2000).

The present study captures pricing problems in terms of double marginalization but goes one step further by incorporating scheduling problems in the form of route developments and/or frequency choices. The model involves a two-stage game with two carriers who choose their complementary networks in the first stage and prices in the second stage. Each carrier's network involves one or two routes that are distributed geographically or distributed in time. If both carriers maintain two routes, then transfer passengers can choose between two alternative connections which they consider as imperfect substitutes. There are only transfer passengers, that is, non-stop passengers are absent. Maintaining a route is costly.

The study develops detailed insights on the relationship between carrier networks and the substitutability of the two alternative connections determined by a differentiation parameter which is part of the standard Dixit (1979) type of utility function. The analysis reveals that carrier collaboration and antitrust immunity can not only eliminate double marginalization but can further create additional incentives to maintain two connections rather than one connection, both of which increases social welfare, depending on the value of the differentiation parameter.

To see that differentiation matters, consider the case of connections that are perfect substitutes. In this case, maintaining two routes can never, that is, neither for a profit-maximizing carrier nor for a welfare-maximizing carrier, be optimal because it increases maintenance costs without creating additional demands and benefits, respectively. The critical level of differentiation between routes that needs to be reached so that independent carriers would maintain two routes in (the subgame-perfect) equilibrium is, however, higher than the corresponding level for collaborating carriers with antitrust immunity. In this sense, collaboration can create stronger incentives to maintain two connections. However, analytical results indicate that the scope for the improvement of carrier networks can be rather limited relative to the social desirability of more complete carrier networks. A possible policy lesson is that airlines should be granted antitrust immunity conditional on network expansion.

Collaboration can increase the incentives to maintain a more extensive and complete network 
also through another channel. Even though the two routes would be sufficiently differentiated to support equilibrium networks with two connections, the perfect complementarity of carrier networks leads to the existence of a coordination problem in the sense that maintaining only one connection always represents an equilibrium network solution. Carrier collaboration can, therefore, support the maintenance of two connections by overcoming the potential coordination problem that exists in the case of independent network choices.

Bilotkach (2019) surveys papers on airline partnerships and antitrust immunity and distinguishes between studies which concentrate on price effects and studies which also consider non-price product characteristics. This paper contributes to the second type by analyzing how carrier collaboration can change network choices and how this can increase welfare depending on the heterogeneity of alternative connections. The paper most closely related to the present study is the one by Czerny et al. (2016). They consider a sequential structure in which the carriers choose frequencies and aircraft sizes in the first stage and air fares in the second stage. They find that independent carriers strategically reduce frequency supply in the first stage in order to lower the other carrier's equilibrium fare in the second stage. In this case, carrier collaboration can increase frequency supply by eliminating the strategic reduction of frequencies by independent carriers, which is consistent with the results presented in this study. This is consistent with the findings by Alderighi and Gaggero (2014) who provide empirical evidence for carrier collaboration in the form of alliances to increase flight frequency. The present study contributes to this strand of the literature by highlighting that their is a geographical interpretation of the problem and by analyzing in detail the role of the heterogeneity between connections and the limited potential for collaboration and antitrust immunity to reach the welfare-optimal network structure. The study further contributes by highlighting the existence of a coordination problem.

The present study abstracts away from the issue of coordinating a given number of flights. This problem has been considered in a complementary study by Bilotkach (2007), who analyzes how carrier collaboration can help coordinating a given number of complementary flights. Adler and Hanany (2016) consider price and frequency choices in parallel carrier networks and find that carrier collaboration without antitrust immunicy could be best for passengers. Alderighi and Gaggero (2018) empirically show that alliance membership can lead to increases in flight cancellations. Brueckner and Flores-Fillol (2020) consider endogenous pricing and frequency choices and provide a detailed discussion of how frequency choices can translate into schedule delays when carrier networks are complementary.

Many studies have theoretically and empirically highlighted the effects of carrier collaboration in the form of alliances and codeshares on air fares (for example, Oum et al., 1996; Park, 1997; Park and Zhang, 2000; Brueckner and Whalen, 2000; Brueckner, 2001 and 2003; Bamberger et al., 2004; Brueckner and Pels, 2005; Ito and Lee, 2005 and 2007; Whalen, 2007; Armantier and Richard, 2008; Gayle, 2008; Wan et al., 2009; Brueckner and Proost, 2010; Brueckner et al., 2011; Bilotkach 
and Hüschelrath, 2011, 2012 and 2013; Zou et al., 2011). Bilotkach (2005) and Zhang and Zhang (2006) analyze the rivalry between airline alliances. That carrier collaboration can reduce welfare by increasing air fares for non-stop passengers in complementary networks has been highlighted by Czerny (2009). Gaggero and Bartolini (2012) empirically analyze the determinants of airline alliances and provide insights on the airline incentives to join or form an alliance. The effect of carrier collaboration on the temporal profile of air fares has been studied by Alderighi et al. (2015). See Zhang and Czerny (2012) for a discussion of earlier studies of airline alliances.

This paper is organized as follows. Section 2 presents the model. The demand functions depending on carrier network choices are derived in Section 3. Section 4 uses the demand functions to analyze best responses in terms of network choices and equilibrium network structures when carriers are independent and networks that maximize joint profits when carriers are allied. Section 4 further analyzes the effects of carrier collaboration on consumer surplus and welfare. Section 5 concludes and develops avenues for future research.

\section{The Model}

The model developed in this section, can have a geographical and a frequency interpretation. Consider the geographical interpretation first. This interpretation involves four airports denoted by $A, H, J$, and $B$. Passengers wish to fly from airports $A$ to $B$ and from $B$ to $A$. There are two carriers denoted by 1 and 2. Each carrier maintains one or two connections. For bilateral agreement reasons, carrier 1 can only maintain links between airports $A$ and $H$ and $A$ and $J$, whereas carrier 2 can only maintain links between airports $B$ and $H$ and $B$ and $J$. Thus, passenger flows between airports $A$ and $B$ require the two carriers to provide complementary links between these two airports. Hence, only when they both fly to airport $J$ or when they both fly to airport $H$, then and only then they can serve passenger demand for flights between $A$ and $B$. Networks in which transfers would not be feasible, for instance, carrier 1 maintains link $A$ and $J$ and carrier 2 maintains link $H$ and $B$, are not considered in order to concentrate on airline networks in which transfer passengers exist. For the same reason, non-stop passengers are absent in the present study. The cost of maintaining a link by, for instance, establishing a ground team at an airport is given by $F$. Per passenger-operating costs and airport charges are normalized to zero.

The three types of combinations of airline networks considered in the analysis are illustrated by Figure 1. Carrier 1's network is indicated by solid lines whereas carrier 2's network is indicated by dashed lines. Part (a) illustrates the full network in which each carrier maintains the maximum of two connections. In this case, passengers can choose whether to transfer flights at airports $H$ or $J$. Part (b) illustrates a set of asymmetric carrier networks in which transfer is possible at airport $H$ only. Part (c) illustrates a set of symmetric carrier networks in which transfer is possible at airport $H$ only. 
(a)

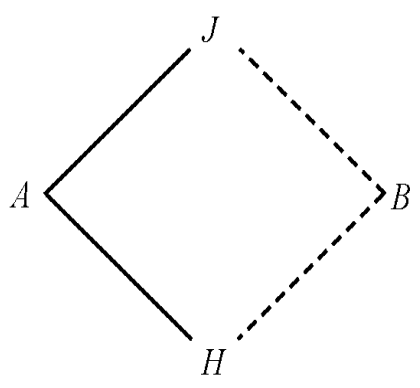

(b)

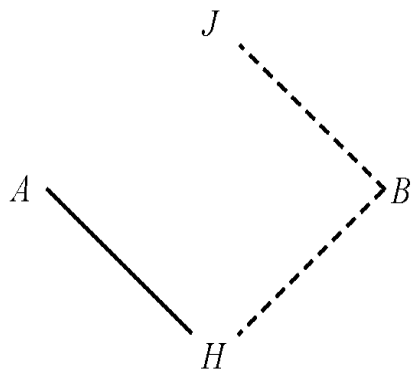

(c)

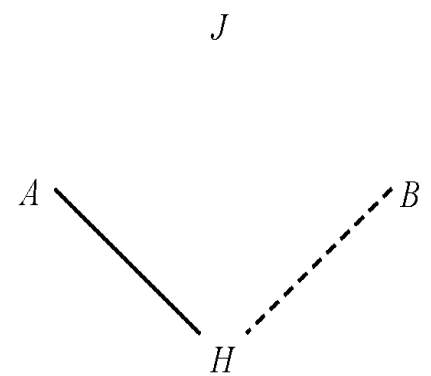

Figure 1: Carrier 1's network is indicated by solid lines, whereas carrier 2's network is indicated by dashed lines. Part (a): Symmetric set of carrier networks in which transfer is possible at airports $H$ and $J$. Part (b): Asymmetric set of carrier networks in which transfer is possible at airport $H$ only. Part (c): Symmetric set of carrier networks in which transfer is possible at airport $H$ only.

Consider the frequency interpretation of this framework. This interpretation involves only one transfer airport but one or two links distributed in time (for instance, flights at different times of the day or flights at different days of the week). An increase in the number of links therefore represents an increase in flight frequency which reduces deviations of the passengers' preferred from the actual flight times and, thus, schedule delays (e.g., Miller, 1972; Douglas and Miller, 1974). The following analysis will concentrate on the geographical interpretation although the approach is more general in the sense that it allows for other interpretations such as flight frequencies and therefore the results are more general than the geographical interpretation would suggest.

Conditional on maintaining the links between airports $i$ and $j$ with $i, j=A, H, J, B$ and $j \neq i$, carriers charge prices denoted by $p_{i j}$ for their services. Denote the quantity of transfer passengers who transfer at airport $H$ by $q_{H}$ and the quantity of transfer passengers who transfer at airport $J$ by $q_{J}$. Passenger benefits, denoted by $B$, are of the Dixit (1979) type and given by

$$
B\left(q_{H}, q_{J}\right)=a \cdot\left(q_{H}+q_{J}\right)-\frac{b}{2} \cdot\left(q_{H}^{2}+q_{J}^{2}\right)-d \cdot q_{H} q_{J}
$$

with $a, b, d, b-d>0$. Considering positive values of the differentiation-parameter $d$ implies that passengers consider transfers at airports $H$ and $J$ as imperfect substitutes. $b-d>0$ implies that the cross-derivative $\partial^{2} B / \partial q_{H} \partial q_{J}=-d$ is smaller in absolute values than the second derivatives $\partial^{2} B / \partial q_{i}=-b$ for $i=H, J$, which ensures the strict concavity of the benefit function in (1).

The differentiation-parameter $d$ is the parameter of interest, which will be used to derive a better understanding of how airline alliances can affect (complementary) networks in the case of substitute connections. To do this, the following compares the outcomes of scenarios with independent and allied carriers. The scenario of independent carriers is modelled as a two-stage game. In the first stage, carriers choose whether to maintain one or two links. In the second stage, carriers choose their 
prices for the maintained links to maximize their individual profits. In the scenario with allied carriers, two carriers simultaneously choose their prices and links so that their joint profit is maximized.

\section{Demand functions}

Let $\delta_{i}$ denote indicator variables that take a value of one (that is, $\delta_{i}=1$ ) if carrier $i$ maintains one link and value two if each of two carriers maintains two links, that is, $\delta_{i}=1,2$.

Symmetric case with two connections. Consider the case in which $\delta_{1}=\delta_{2}=2$ and, thus, passengers can choose between transferring at airports $H$ or $J$. To economize notation, let $p_{H}$ denote the sum of prices paid by passengers who transfer at airport $H$, that is, $p_{H}=p_{A H}+p_{H B}$ and let $p_{J}$ denote the sum of prices paid by passengers who transfer at airport $J$, that is, $p_{J}=p_{A J}+p_{J B}$. In this case, the demands are determined by the equilibrium conditions

$$
\frac{\partial B\left(q_{H}, q_{J}\right)}{\partial q_{H}}-p_{H}=a-b q_{H}-d q_{J}-p_{H}=0
$$

and

$$
\frac{\partial B\left(q_{H}, q_{J}\right)}{\partial q_{J}}-p_{J}=a-b q_{J}-d q_{H}-p_{J}=0 .
$$

These conditions ensure that passengers will transfer at airports $H$ and $J$ if their benefits from traveling is not lower than the prices they have to pay for the two connections. Simultaneously solving the two equilibrium conditions in (2) and (3) yields the demand for transfer passengers at airport $H$, denoted by $D_{H}\left(p_{H}, p_{J} ; \delta_{1}, \delta_{2}\right)$, and the demand for transfer passengers at airport $J$, denoted by $D_{J}\left(p_{H}, p_{J} ; \delta_{1}, \delta_{2}\right)$. For $\delta_{1}=\delta_{2}=2$, demands can be written as

$$
D_{H}\left(p_{H}, p_{J} ; 2,2\right)=\frac{1}{b^{2}-d^{2}} \cdot\left(a(b-d)-b p_{H}+d p_{J}\right)
$$

and

$$
D_{J}\left(p_{H}, p_{J} ; 2,2\right)=\frac{1}{b^{2}-d^{2}} \cdot\left(a(b-d)-b p_{J}+d p_{H}\right)
$$

as long as prices are low enough to ensure that demands are non-negative. These demand functions imply that the demand for transfer passengers at airport $i$ are decreasing in the price $p_{i}$ with $i=H, J$ and increasing in the price $p_{j}$ with $j \neq i$. The demand functions further imply that a simultaneous increase in prices $p_{H}$ and $p_{J}$ by the same amount reduces the transfer passengers' demands at both airports $H$ and $J$.

Symmetric case with one connection. Consider the case in which $\delta_{1}=\delta_{2}=1$ and, thus, passengers can only transfer at airport $H$ or airport $J$. Without loss of generality, assume that, in this case, passengers transfer at airport $H$. In this case, the demand $D_{H}\left(p_{H}, p_{J} ; 1,1\right)$ is determined by the equilibrium condition

$$
\frac{\partial B\left(q_{H}, 0\right)}{\partial q_{H}}-p_{H}=a-b q_{H}-p_{H}=0
$$


leading to

$$
D_{H}\left(p_{H}, p_{J} ; 1,1\right)=\frac{a-p_{H}}{b}
$$

in which the right-hand side is decreasing in the price $p_{H}$ for $p_{H} \leq a$.

Asymmetric case. Consider the case in which $\delta_{j} \neq \delta_{i}$ with $j \neq i$. This means that one carrier maintains one link whereas the other carrier maintains two links. Without loss of generality, assume that, in this case, carrier 1 maintains one link between $A$ and $H$ with $\delta_{1}=1$ whereas carrier 2 maintains two links with $\delta_{2}=2$. Despite that carrier 2 maintains two links, the perfect complementarity of carrier networks implies that passengers can only transfer at airport $H$ because there is no service between airports $A$ and $J$ provided by carrier 1 . Therefore, the demand function in the present asymmetric case can be described by the demand function which exists in the symmetric case with one feasible connection involving airport $H$, that is, $D_{H}\left(p_{H}, p_{J} ; 1,2\right)=D_{H}\left(p_{H}, p_{J} ; 1,1\right)$.

\section{Equilibrium networks}

Carrier 1's profit, denoted by $\pi_{1}$, depending on the number of passengers transferring at airports $H$ and $J$, can be written as

$$
\pi_{1}\left(p_{H}, p_{J} ; \delta_{1}, \delta_{2}\right)=p_{A H} \cdot D_{H}\left(p_{H}, p_{J} ; \delta_{1}, \delta_{2}\right)+p_{A J} \cdot D_{H}\left(p_{H}, p_{J} ; \delta_{1}, \delta_{2}\right)-\delta_{1} F
$$

The first term on the right-hand side represents carrier 1's revenue from passengers transferring at airport $H$, whereas the second term represents the corresponding revenue from passengers transferring at airport $J$. The third term on the right-hand side represents the network maintenance costs, which can be equal to $F$ or $2 F$ depending on whether a single connection or two connections are maintained, respectively. Carrier 2's profit, denoted by $\pi_{2}$, depending on the number of passengers transferring at airports $H$ and $J$, can analogously be written as

$$
\pi_{2}\left(p_{H}, p_{J} ; \delta_{1}, \delta_{2}\right)=p_{H B} \cdot D_{H}\left(p_{H}, p_{J} ; \delta_{1}, \delta_{2}\right)+p_{J B} \cdot D_{H}\left(p_{H}, p_{J} ; \delta_{1}, \delta_{2}\right)-\delta_{2} F .
$$

These profit functions can be used to derive the prices and networks in the subgame-perfect equilibrium when there are two independent carriers as well as the profit-maximizing set of prices and network when carriers form an alliance.

\subsection{Independent carriers}

Two connections. Consider the case of independent carriers in which both carriers maintain two links, that is, $\delta_{1}=\delta_{2}=2$. Without loss of generality, the following concentrates on carrier 1. Carrier 1 's profit depending on prices can be written as

$$
\begin{aligned}
\pi_{1}\left(p_{H}, p_{J} ; 2,2\right) & =p_{A H} \cdot D_{H}\left(p_{H}, p_{J} ; 2,2\right)+p_{A J} \cdot D_{J}\left(p_{H}, p_{J} ; 2,2\right)-2 F \\
& =\frac{1}{b^{2}-d^{2}}\left(\left(p_{A H}+p_{A J}\right) a(b-d)-p_{A H}\left(b p_{H}-d p_{J}\right)-p_{A J}\left(b p_{J}-d p_{H}\right)\right)-2 F
\end{aligned}
$$


Carrier 1's best responses in terms of prices $p_{A i}$, indicated by superscript $b r$ (hereafter best responses will be indicated by superscript $b r$ ), are determined by the first-order conditions

$$
\begin{aligned}
\frac{\partial \pi_{1}\left(p_{H}, p_{J} ; 2,2\right)}{\partial p_{A i}} & =D_{i}\left(p_{H}, p_{J} ; 2,2\right)+p_{A i} \cdot \frac{\partial D_{i}\left(p_{H}, p_{J} ; 2,2\right)}{\partial p_{i}}+p_{A j} \cdot \frac{\partial D_{j}\left(p_{H}, p_{J} ; 2,2\right)}{\partial p_{i}} \\
& =\frac{1}{b^{2}-d^{2}} \cdot\left(a(b-d)-b \cdot\left(2 p_{A i}^{b r}+p_{i B}\right)+d \cdot\left(2 p_{A j}+p_{j B}\right)\right)=0
\end{aligned}
$$

with $i=H, J$ and $j \neq i$. The third term on the right-hand side of (11a) exists and is positive in sign because transfer airports are considered as substitutes by passengers. This term indicates that carriers' best responses consider the positive effect of an increase in the charge on one link on the revenue obtained from maintaining the other link. Simultaneously solving the first-order conditions for carrier 1 in (11b) for $i=H, J$ yields best responses

$$
p_{A i}^{b r}\left(p_{i B}\right)=\frac{a-p_{i B}}{2}
$$

for $i=H, J$. Best responses are independent of the differentiation-parameter and of the prices charged at the other connection and they are decreasing in the other carrier's price for complementary link on the same connection; thus, carrier prices on the same connection are strategic substitutes in the sense described by Bulow et al. (1985). Best responses are independent of the differentiation parameter because carriers internalize the effect of prices on the profits that can be obtained on the other connection. Using symmetry, letting $N$ denote the equilibrium prices (henceforth, equilibrium prices will always be indicated by superscript $N$ ) and solving for equilibrium prices leads to

$$
p_{A i}^{N}=p_{i B}^{N}=\frac{a}{3}
$$

for $i=H, J$. This yields the well-known result that the sum of equilibrium prices, $p_{A i}^{N}+p_{i B}^{N}=2 a / 3$, exceeds monopoly price, which is equal to $a / 2$. Equilibrium prices are further independent of the differentiation-parameter because best responses are independent of the differentiation-parameter. Using equilibrium prices in (13) and letting $p_{i}^{N}$ denote the sum of equilibrium prices $p_{A i}^{N}+p_{i B}^{N}$, equilibrium profits can be written as

$$
\pi_{1}\left(p_{H}^{N}, p_{J}^{N} ; 2,2\right)=\frac{2 a^{2}}{9(b+d)}-2 F .
$$

Whereas best responses and equilibrium prices are independent of the differentiation-parameter, equilibrium profits are decreasing in the differentiation-parameter, which is an artefact of the Dixit type of benefit functions because this functional form implies that an increase in the differentiation parameter reduces total market size. ${ }^{1}$

\footnotetext{
${ }^{1}$ To see this, consider the demand functions in (4) and (5). Using symmetry in the sense that $p_{H}=p_{J}$, demand for passengers transfer at airport $i$ can be written as $D_{i}=\left(a-p_{i}\right) /(b+d)$. This shows that market size is indeed decreasing in the differentiation parameter $d$. Levitan and Shubik (1971) provided a different demand functional form with an adjusted slope so that the aggregate demand is independent of the number of products or services in the market when prices are all equal. See Vives (1999) for a more detailed discussion on this funcitonal form. However, Levitan and Shubik's (1971) functional form does not include a differentiation-parameter that is independent of the number of products. Therefore, it is not considered in our analysis.
} 
One connection. Consider the case of independent carriers in which both carriers maintain only one link and the two links are connected. By assumption, passengers travel at airport $H$. Carrier 1's profit depending on prices can be written as

$$
\begin{aligned}
\pi_{1}\left(p_{H} ; 1,1\right) & =p_{A H} \cdot D_{H}\left(p_{H} ; 1,1\right)-F \\
& =p_{A H} \frac{a-p_{H}}{b}-F
\end{aligned}
$$

The first term on the right-hand side of $(15 \mathrm{a})$ shows that in the presence of one connection, there is only one source of revenue whereas the second term shows that the maintenance cost is counted once. Carrier 1's best response in terms of the price $p_{A H}$ is determined by the first-order condition

$$
\begin{aligned}
\frac{\partial \pi_{1}\left(p_{H} ; 1,1\right)}{\partial p_{A H}} & =D_{H}\left(p_{H} ; 1,1\right)+p_{A H} \cdot \frac{\partial D_{H}\left(p_{H} ; 1,1\right)}{\partial p_{A H}} \\
& =\frac{1}{b}\left(a-2 p_{A H}^{b r}-p_{H B}\right)=0 .
\end{aligned}
$$

Solving the first-order condition for carrier 1 in (16b) yields the best response

$$
p_{A H}^{b r}\left(p_{H B}\right)=\frac{a-p_{H B}}{2} .
$$

Thus, best response functions under one connection are identical to the best response functions for each connection in the case of two connections. Best responses are still decreasing in the other carrier's price and therefore carrier prices are still strategic substitutes. Using symmetry and solving yields the equilibrium prices

$$
p_{A H}^{N}=p_{H B}^{N}=\frac{a}{3} .
$$

Again, the equilibrium prices under one connection are identical to the equilibrium prices for each link under two connections. This is not surprising given that the best responses are identical under one and two connections. Using equilibrium prices in (18), equilibrium profits can be written as

$$
\pi_{1}\left(p_{H}^{N}, ; 1,1\right)=\frac{a^{2}}{9 b}-F
$$

Comparing the first terms on the right-hand sides of (14) and (19) shows that the average equilibrium revenue in the case of two connections is smaller than the equilibrium revenue in the case of one connection because the differentiation-parameter $d$ is positive by assumption. This implies that the presence of the differentiation-parameter reduces the airlines' revenues by reducing the market size whereas it has no impact on the equilibrium prices. However, the total revenue still increases in the case of two connections if the differentiation-parameter is small enough.

Asymmetric case. Consider the case of independent carriers in which carrier 1 maintains only one connection whereas carrier 2 maintains two connections. The perfect complementarity of carrier networks implies that there is no passenger transferring at airport $J$ because there is no service between airports $A$ and $J$ provided by carrier 1 . Passengers can only transfer at airport $H$, and demand in 
the asymmetric case is identical to the demand in the symmetric case with one connection, that is, $D_{H}\left(p_{H}, p_{J} ; 1,2\right)=D_{H}\left(p_{H}, p_{J} ; 1,1\right)$. Therefore, equilibrium prices in the asymmetric case are identical to the equilibrium prices in (18), which exist when both carriers maintain one connection, and the revenues are identical for these two cases too. The only difference between these two cases is that carrier 2 maintains one more connection between airports $J$ and $B$ and, thus, pays one more unit of maintenance cost given by $F$.

Equilibrium networks. In the asymmetric case, there is no profit for carrier 2 to maintain the connection between airports $J$ and $B$ with a maintenance cost $F$. This is because there is no transfer passengers transferring at airport $J$ in this case because of the perfect complementarity between carrier networks. This implies that maintaining two connections given that the other carrier maintains only one connection can never be a best response. Equilibrium networks will therefore be derived by comparing carrier profits in the cases in which both cases either offer one or two connections.

The following assumption sets an upper limit on the maintenance costs $F$, which is to ensure that equilibrium profits are positive.

Assumption 1 Maintenance costs are sufficiently low in the sense that

$$
F<\frac{a^{2}}{9(b+d)}
$$

An increase in the maximum reservation price $a$ increases the market size whereas increases in the slope parameters $b$ and $d$ reduce market size. The right-hand side is therefore increasing in market size because it increases in $a$ and decreases in $b$ and $d$, which is intuitive. Assumption 1 will henceforth be assumed to hold in the entire subsequent analysis.

Comparing equilibrium profits in (14) and (19) reveals:

Lemma 1 Consider independent carriers. There is a critical value of the differentiation parameter, denoted by $d^{I}$ with

$$
d^{I} \equiv \frac{b\left(a^{2}-9 b F\right)}{a^{2}+9 b F},
$$

such that if $d>d^{I}$, then there exists one subgame-perfect Nash equilibrium in which both carriers maintain only one link, that is, $\delta_{1}=\delta_{2}=1$, whereas if $d \leq d^{I}$, then there exist two subgame-perfect Nash equilibria in which both carriers either maintain only one link, that is, $\delta_{1}=\delta_{2}=1$, or both carriers maintain two links, that is, $\delta_{1}=\delta_{2}=2$ in which the latter Pareto dominates the former.

The perfect complementarity between carrier networks implies that carriers cannot increase their own profit by unilaterally deviating from one to two connections. Therefore, a situation in which both carriers maintain only one link always represents an equilibrium solution. However, carriers can unilaterally shut down one link and force all passengers to use the one remaining connection, 
which would save maintenance costs. Because equilibrium profits in the case of two connections are decreasing in the differentiation-parameter as shown in (14), maintaining two connections can only be an equilibrium solution if the differentiation-parameter is small enough in the sense that $d \leq d^{I}$. If maintaining the two connections is an equilibrium solution, maintaining two links rather than one link must be Pareto dominant because otherwise it could not be an equilibrium solution because carriers can unilaterally shut down one connection.

\subsection{Allied carriers}

If the two carriers form an alliance, then they behave as one company and simultaneously choose prices and networks to maximize their joint profit. This reflects a situation in which carriers are granted antitrust immunity. In reality, many carriers are granted antitrust immunity in the case of complementary networks of the types displayed in Figure 1 . The total profits are denoted by $\pi$ with $\pi=\pi_{1}+\pi_{2}$. For $\delta_{1}=\delta_{2}=2$ and $\delta_{1}=\delta_{2}=1$, total profits can be written as

$$
\begin{aligned}
\pi\left(p_{H}, p_{J} ; 2,2\right) & =p_{H} \cdot D_{H}\left(p_{H}, p_{J} ; 2,2\right)+p_{J} \cdot D_{J}\left(p_{H}, p_{J} ; 2,2\right)-4 F \\
& =\frac{1}{b^{2}-d^{2}}\left(a(b-d)\left(p_{H}+p_{J}\right)-b\left(p_{H}^{2}+p_{J}^{2}\right)+2 d p_{H} p_{J}\right)-4 F
\end{aligned}
$$

and

$$
\begin{aligned}
\pi\left(p_{H} ; 1,1\right) & =p_{H} \cdot D_{H}\left(p_{H} ; 1,1\right)-2 F \\
& =p_{H} \frac{a-p_{H}}{b}-2 F
\end{aligned}
$$

respectively.

Allied carriers maximize their total profit by the choice of the (total) prices $p_{H}$ and $p_{J}$. Let superscript $A$ denote the profit-maximizing prices of allied carriers. In the case of two connections, allied prices are determined by the first-order conditions

$$
\begin{aligned}
\frac{\partial \pi\left(p_{H}^{A}, p_{J}^{A} ; 2,2\right)}{\partial p_{H}} & =\frac{\partial \pi\left(p_{H}^{A}, p_{J}^{A} ; 2,2\right)}{\partial p_{J}} \\
& =D_{i}\left(p_{H}^{A}, p_{J}^{A} ; 2,2\right)+p_{i}^{A} \cdot \frac{\partial D_{i}\left(p_{H}^{A}, p_{J}^{A} ; 2,2\right)}{\partial p_{i}}+p_{j}^{A} \cdot \frac{\partial D_{j}\left(p_{H}^{A}, p_{J}^{A} ; 2,2\right)}{\partial p_{i}} \\
& =\frac{1}{b^{2}-d^{2}} \cdot\left(a(b-d)-2 b p_{i}^{A}-(b-d) p_{j}^{A}\right)=0
\end{aligned}
$$

with $i=H, J$ and $j \neq i$. Comparing the second and third terms on the right-hand sides of (11a) and (24b), respectively, shows that allied carriers consider each other's profit rather than only their own as would be the case with independent carriers, which eliminates double marginalization. This is because the prices $p_{i}$ and $p_{j}$ reflect to the total prices rather than the individual carriers' prices.

In the case of one connection, the allied price is determined by the first-order condition

$$
\begin{aligned}
\frac{\partial \pi\left(p_{H}^{A} ; 1,1\right)}{\partial p_{H}} & =D_{H}\left(p_{H}^{A} ; 1,1\right)+p_{H}^{A} \cdot \frac{\partial D_{H}\left(p_{H}^{A} ; 1,1\right)}{\partial p_{H}} \\
& =\frac{a-2 p_{H}^{A}}{b}=0 .
\end{aligned}
$$


Comparing the second terms on the right-hand sides of (16a) and (25a) shows again that allied carriers consider each other's profit rather than only their own as would be the case with independent carriers, which eliminates double marginalization.

Simultaneously solving the first-order conditions yields the profit-maximizing (total) prices of allied carriers, which are given by

$$
p_{i}^{A}=\frac{a}{2}
$$

for $\delta_{1}=\delta_{2}=1$ and $\delta_{1}=\delta_{2}=2$. Prices are independent of the network structure because the monopoly prices are determined by the semi-price elasticities of the demand functions, which depend on the maximum-reservation price $a$ but not the demand slopes. Using these profit-maximizing prices, total profits in the cases of two connections and one connection can be written as

$$
\pi\left(p_{H}^{A}, p_{J}^{A} ; 2,2\right)=\frac{a^{2}}{2(b+d)}-4 F
$$

and

$$
\pi\left(p_{H}^{A} ; 1,1\right)=\frac{a^{2}}{4 b}-2 F,
$$

respectively. Comparing the right-hand sides in (27) and (28) shows that the average equilibrium profit in the case of two connections is smaller than the equilibrium profit in the case of one connection because the differentiation-parameter $d$ is positive by assumption. This implies that the presence of the differentiation-parameter reduces the airline's profits only by reducing the market size because it has no impact on the equilibrium prices.

Under Assumption 1, $F<\frac{a^{2}}{9(b+d)}$ and thus $F<\frac{a^{2}}{8(b+d)}$ implying that the right-hand side of $(27)$ is strictly positive. Therefore, Assumption 1 ensures that equilibrium profits are positive both in the cases of independent and allied carriers.

Comparing equilibrium profits in (27) and (28) reveals:

Lemma 2 Consider allied carriers. There is a critical value of the differentiation-parameter, denoted by $d^{A}$ with

$$
d^{A} \equiv \frac{b\left(a^{2}-8 b F\right)}{a^{2}+8 b F}
$$

and $d^{A}>d^{I}$, such that if the differentiation-parameter is large enough in the sense that $d \geq d^{A}$, then carriers maintain one connection, whereas if the differentiation-parameter is small enough in the sense that $d \leq d^{A}$, then carriers maintain two connections.

Lemma 2 shows that the critical value of the differentiation-parameter in the case of allied carriers is larger than that in the case of independent carriers, that is, $d^{A}>d^{I}$. This means that the allied carriers are more inclined to maintain two connections than the independent carriers. This is because the allied carriers are collaborating and thereby eliminate double marginalization, which helps to better exploit the revenue opportunities arising from the maintenance of two connections relative to the revenues that could be obtained from maintaining only one connection. 
Comparing the profits with allied carriers and independent carriers in the case of two connections and one connection respectively shows that profits with alliance are always higher than the profits with independent carriers, which is almost straightforward because of the elimination of double marginalization in the presence of allied carriers. The network implications are more complex and can be described as follows:

Proposition 1 The relationship between the networks of independent or allied carriers can be characterized as:

(i) If $d \leq d^{I}$, there is a coordination problem in the sense that the networks of allied carriers may or may not be identical to the equilibrium networks of independent carriers.

(ii) If $d \in\left(d^{I}, d^{A}\right)$, then there is a discrepancy between the networks of allied carriers and equilibrium networks of independent carriers in the sense that allied carriers will maintain two connections whereas independent carriers will maintain only one connection in equilibrium. For all other values of the differentiation parameter, that is, $d \notin\left(d^{I}, d^{A}\right)$, the networks of allied carriers are identical to the equilibrium networks of independent carriers.

(iii) If $d \geq d^{I}$, the networks of allied carriers are identical to the equilibrium networks of independent carriers.

Proposition 1 shows that allied carriers can change networks in two ways relative to independent carriers. First, because, $d^{I}<d^{A}$, the presence of allied stimulates the maintenance of the twoconnection network. This theoretical finding is consistent with the results derived by Czerny et al. (2016) and the empirical findings by Alderighi and Gaggero (2014). Second, for $d>d^{I}$ allied can solve the coordination problem of independent carriers because independent carriers may stick to the one-connection network in equilibrium despite the fact that the two-connection network would be more profitable for them. This is because of the absence of a unique subgame-perfect equilibrium under this parameter condition.

\subsection{Consumer surplus and welfare implications}

Passengers only pay a ticket price, that is, there is no congestion costs or other costs involved. Therefore, consumer surplus, denoted by $C S$, is the difference between the benefits of travelling and the total payment to the airlines and given by

$$
C S\left(q_{H}, q_{J}\right)=B\left(q_{H}, q_{J}\right)-p_{H} q_{H}-p_{J} q_{J}
$$


Substituting passenger quantities by their demand functions yields the consumer surplus depending on prices and networks, which can be written as

$$
\begin{aligned}
C S\left(p_{H}, p_{J} ; \delta_{1}, \delta_{2}\right)= & B\left(D_{H}\left(p_{H}, p_{J} ; \delta_{1}, \delta_{2}\right), D_{J}\left(p_{H}, p_{J} ; \delta_{1}, \delta_{2}\right)\right) \\
& -p_{H} \cdot D_{H}\left(p_{H}, p_{J} ; \delta_{1}, \delta_{2}\right)-p_{J} \cdot D_{J}\left(p_{H}, p_{J} ; \delta_{1}, \delta_{2}\right) .
\end{aligned}
$$

Concentrating on the symmetric cases with $\delta_{1}=\delta_{2}=2$ and $\delta_{1}=\delta_{2}=1$, consumer surplus depending on prices can be written as

$$
C S\left(p_{H}, p_{J} ; 2,2\right)=\frac{1}{2\left(b^{2}-d^{2}\right)}\left(2 a^{2}(b-d)-2 a(b-d)\left(p_{H}+p_{J}\right)+b\left(p_{H}^{2}+p_{J}^{2}\right)-2 d p_{H} p_{J}\right)
$$

and

$$
C S\left(p_{H} ; 1,1\right)=\frac{1}{2 b}\left(a-p_{H}\right)^{2},
$$

respectively. For given prices, passengers always prefer to have two connections relative to one connection because it provides them with the possibility to choose between two differentiated services whereas such a choice would be absent if carriers maintain only one connection. This directly implies:

Lemma 3 For any given set of prices $p_{H}$ and $p_{J}$, consumer surplus is always higher when carriers maintain two connection than when they maintain one connection, that is, $C S\left(p_{H}, p_{J} ; 2,2\right) \geq$ $C S\left(p_{H} ; 1,1\right)$.

Operating costs and airport charges are normalized to zero by assumption. The only cost is a fixed maintenance cost for each connection. Let $W$ denote the welfare, which is the sum of consumer surplus and total profit and can be written as

$$
\begin{aligned}
W\left(p_{H}, p_{J} ; \delta_{1}, \delta_{2}\right) & =C S\left(p_{H}, p_{J} ; \delta_{1}, \delta_{2}\right)+\pi\left(p_{H}, p_{J} ; \delta_{1}, \delta_{2}\right) \\
& =B\left(D_{H}\left(p_{H}, p_{J} ; \delta_{1}, \delta_{2}\right), D_{J}\left(p_{H}, p_{J} ; \delta_{1}, \delta_{2}\right)\right)-\left(\delta_{1}+\delta_{2}\right) F
\end{aligned}
$$

Welfares in the cases of two connections and one connection can be written as

$$
W\left(p_{H}, p_{J} ; 2,2\right)=\frac{1}{2\left(b^{2}-d^{2}\right)}\left(2 a^{2}(b-d)-b\left(p_{H}^{2}+p_{J}^{2}\right)+2 d p_{H} p_{J}\right)-4 F
$$

and

$$
W\left(p_{H} ; 1,1\right)=\frac{1}{2 b}\left(a^{2}-p_{H}^{2}\right)-2 F
$$

respectively.

Because passenger costs are normalized to zero, the first-best welfare is obtained when the ticket price is zero. The reason is that any positive ticket price will discourage passengers from traveling although their benefits from traveling would be positive. The first-best welfare in the cases of two connections and one connection can be written as

$$
W(0,0 ; 2,2)=\frac{a^{2}}{b+d}-2 F
$$


and

$$
W(0 ; 1,1)=\frac{a^{2}}{2 b}-F
$$

respectively.

Comparing first-best welfare in (37) and (38) reveals:

Lemma 4 There is a critical value of the differentiation-parameter, denoted by $d^{W}$ with

$$
d^{W}=\frac{b\left(a^{2}-2 b F\right)}{a^{2}+2 b F}
$$

and $d^{W}>d^{A}>d^{I}$, such that if the differentiation-parameter is large enough in the sense that $d>d^{W}$, then welfare in the case of one connection is higher than in the case of two connections, whereas if the differentiation-parameter is small enough in the sense that $d \leq d^{W}$, then welfare in the case of one connection is lower than in the case of two connections.

The first-best welfare in the case of two connections is decreasing in the differentiation-parameter as shown in (37). This implies that if the differentiation-parameter is high in the sense that $d \geq d^{W}$, the extra welfare achieved by maintaining the second connection will be equal to or even smaller than the extra cost in terms of maintenance costs. This means that maintaining two connections reduces the total welfare relative to maintaining only one connection under these parameter conditions. It is therefore not useful from the welfare viewpoint to maintain two connections if these connections are close substitutes. The welfare gain from enabling passengers to choose their preferred is small relative to the maintenance costs in this case.

Consider the total welfare for independent carriers in the case of two connections and one connection. Using the equilibrium prices $p_{H}^{N}=p_{J}^{N}=p_{A i}^{N}+p_{i B}^{N}=2 a / 3$, which are identical in the case of two connections and one connection, the equilibrium welfares $W\left(p_{H}^{N}, p_{J}^{N} ; 2,2\right)$ and $W\left(p_{H}^{N} ; 1,1\right)$ can be written as

$$
W\left(p_{H}^{N}, p_{J}^{N} ; 2,2\right)=\frac{10 a^{2}}{9(b+d)}-4 F
$$

and

$$
W\left(p_{H}^{N} ; 1,1\right)=\frac{5 a^{2}}{9 b}-2 F,
$$

respectively. Given Assumption 1 , both $W\left(p_{H}^{N}, p_{J}^{N} ; 2,2\right)$ and $W\left(p_{H}^{N} ; 1,1\right)$ are positive. Comparing equilibrium welfares in (40) and (41) reveals:

Lemma 5 Consider independent carriers. There is a critical value of the differentiation parameter, denoted by $d^{I W}$ with

$$
d^{I W} \equiv \frac{b\left(5 a^{2}-18 b F\right)}{5 a^{2}+18 b F}
$$

$d^{I W}>d^{A}>d^{I}$, and $d^{I W}<d^{W}$, such that if the differentiation-parameter is large enough in the sense that $d>d^{I W}$, then welfare in the case of one connection is higher than in the case of two connections, 


\begin{tabular}{cccc} 
Objective function & Independent carriers & Allied carriers & First-best \\
\hline Profit & $d^{I}$ & $d^{A}$ & - \\
Welfare & $d^{I W}$ & $d^{A W}$ & $d^{W}$ \\
\hline
\end{tabular}

Table 1: Summary of the critical values of differentiation-parameter.

whereas if the differentiation-parameter is small enough in the sense that $d \leq d^{I W}$, then welfare in the case of two connections is higher than in the case of one connection.

Consider the welfare for allied carriers in the case of two connections and one connection. Using the profit-maximizing prices $p_{H}^{A}=p_{J}^{A}=a / 2$, which are identical in the cases of two connections and one connection, the welfares $W\left(p_{H}^{A}, p_{J}^{A} ; 2,2\right)$ and $W\left(p_{H}^{A} ; 1,1\right)$ achieved by allied carriers can be written as

$$
W\left(p_{H}^{A}, p_{J}^{A} ; 2,2\right)=\frac{3 a^{2}}{2(b+d)}-4 F
$$

and

$$
W\left(p_{H}^{A} ; 1,1\right)=\frac{3 a^{2}}{4 b}-2 F,
$$

respectively. Given Assumption 1 , both $W\left(p_{H}^{A}, p_{J}^{A} ; 2,2\right)$ and $W\left(p_{H}^{A} ; 1,1\right)$ are positive. Comparing welfares in (43) and (44) reveals:

Lemma 6 Consider allied carriers. There is a critical value of the differentiation-parameter, denoted by $d^{A W}$ with

$$
d^{A W} \equiv \frac{b\left(3 a^{2}-8 b F\right)}{3 a^{2}+8 b F},
$$

$d^{A W}>d^{I W}>d^{A}>d^{I}$, and $d^{A W}<d^{W}$, such that if the differentiation parameter is large enough in the sense that $d>d^{A W}$, then welfare in the case of two connections is lower than in the case of one connection, whereas if the differentiation parameter is small enough in the sense that $d \leq d^{A W}$, then welfare in the case of two connections is lower than in the case of one connection.

Table 1 summarizes the five critical values of differentiation-parameter in Lemma 1, 2, 4, 5 and 6 . Specifically, Lemma 6 implies the following unambiguous relationships:

$$
d^{I}<d^{A}<d^{I W}<d^{A W}<d^{W}
$$

Thus, if the carriers are indifferent between one or two connections, then the welfare-maximizer prefers two connections in the sense that $d^{I}<d^{I W}$ and $d^{A}<d^{A W}$. This is because prices are identical both in the case of two connections and one connection but consumer surplus is higher in the case of two connections as highlighted by Lemma 3. Altogether,

Proposition 2 From the welfare-viewpoint, both independent and allied carriers are too reluctant to maintain two connections in the sense that $d^{i}<d^{i W}<d^{W}$ for $i=I, A$. 


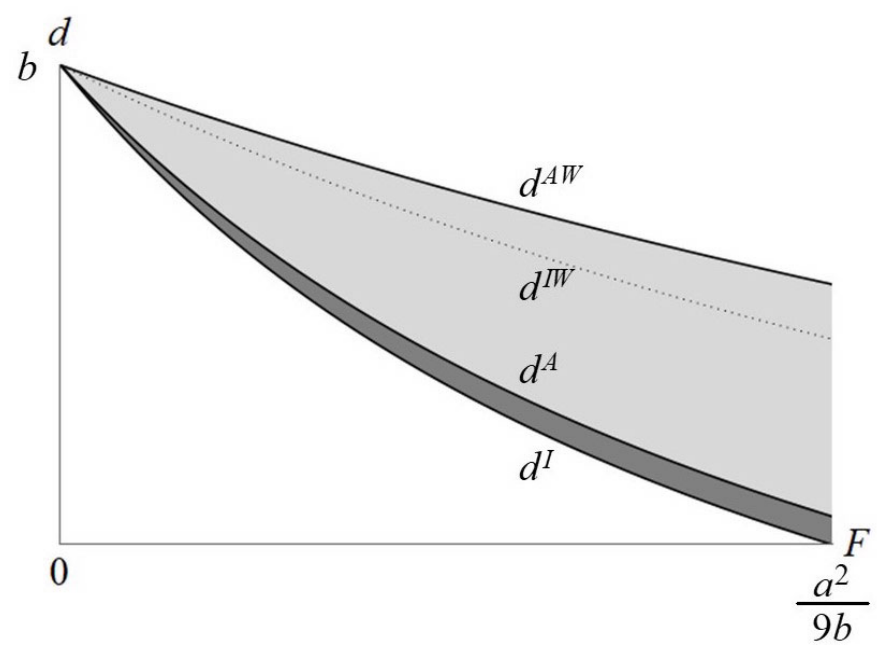

Figure 2: Relationship between the critical value of differentiation-parameter, $d$, and the maintainance cost, $F$

This is an important policy insight from the present study because it highlights that alliances and antitrust immunity can improve carrier networks (and pricing) but that there remains substantial room for further improvements in carrier networks to reach the first-best outcome and optimize pricing and networks from the welfare viewpoint.

To provide a better understanding of the orders of magnitude with respect to the performance of carriers in the case of one and two connections from the welfare-viewpoint, Figure 2 displays the relationship between the critical value of the differentiation-parameter and the maintenance cost. The four curves from top to bottom are $d^{A W}, d^{I W}, d^{A}$ and $d^{I}$, respectively. Specifically, $d^{I W}$ is depicted by a dotted line. The light-gray area on the top captures the difference between $d^{A W}$ and $d^{A}$ and shows the discrepancy between the assessments of carrier networks from the welfare and carriers' viewpoints. Whereas the gray area at the bottom captures the difference between $d^{A}$ and $d^{I}$ and shows the discrepancy between allied carriers that maintain two connections and independent carriers that maintain one connection. The light-gray area is much bigger than the gray area, which is a consistent result given different $a$ and $b$ values. This implies that although allied carriers are more inclined to maintain two connections than independent carriers, the improvement in incentives to maintain two connections is relative minor in the sense that a large areas remain in which there is a discrepancy between the network choices of allied carriers and the corresponding choices from the welfare viewpoint. A policy lesson could be to combine antitrust immunity for allied carriers with services obligations schemes for specific connections that otherwise would remain shut. 


\section{Conclusions}

This study considered two carriers with perfectly complementary networks in which carrier networks maintain only one connection for transfer passengers or two alternative connections. There are only transfer passengers, and if carriers maintain two connections those are considered as imperfect substitutes by passengers. Carriers may act independently or collaborate as in the case of a merger, which is called the case of allied carriers with antitrust immunity. In the case of independent carriers, carriers independently choose their networks in the first stage by anticipating second stage-equilibrium prices.

The study showed that although allied carriers are more inclined to maintain two connections than independent carriers, substantial discrepancies between the network choices of allied carriers and the corresponding choices from the welfare viewpoint remain. The policy lesson is to combine antitrust immunity for allied carriers with services obligations schemes for specific connections that otherwise would remain shut or, considering the frequency interpretation of the framework, frequency obligations schemes to reduce schedule delays.

There are several avenues for future research. One would involve the consideration of overlapping networks. The current study assumes that there is only one carrier maintaining each route, whereas there are many real-world cases in which networks are overlapping in the sense that several carriers are maintaining the same routes in parallel. Another involves the consideration of economies of density (Caves et al., 1984). Higher passenger densities usually allow using, for instance, bigger and more fuel efficient aircraft, which reduces unit costs. If substantial economies of density exist, there is an incentive for carriers to concentrate passengers on certain connection and save maintenance costs by cooperatively using airport facilities even though connections are strongly considered as imperfect substitutes. Finally, a network with non-stop passengers in addition to transfer passengers would be worthwhile investigating.

\section{References}

Adler, N. and Hanany, E., 2016. Regulating inter-firm agreements: The case of airline codesharing in parallel networks. Transportation Research Part B, 31-54.

Alderighi, M. and Gaggero, A., 2014. The effects of global alliances on international flight frequencies: Some evidence from Italy. Journal of Air Transport Management 39, 30-33.

Alderighi, M. and Gaggero, A., 2018. Flight cancellations and airline alliances: Empirical evidence from Europe. Transportation Research Part E 116, 90-101.

Alderighi, M., Gaggero, A. and Piga, C., 2015. The effect of code-share agreements on the temporal profile of airline fares. Transportation Research Part A 79, 42-54. 
Armantier, O. and Richards, O., 2008. Domestic airline alliances and consumer welfare. RAND Journal of Economics 39, 875-904.

Bamberger, G. E., Carlton, D. W. and Neumann, L. R., 2004. An empirical investigation of the competitive effects of domestic airline alliances. Journal of Law and Economics 47, 195-222.

Bilotkach, V., 2005. Price competition between international airline alliances. Journal of Transport Economics and Policy 39, 167-189.

Bilotkach, V., 2007. Airline partnerships and schedule coordination. Journal of Transport Economics and Policy 41, 413-425.

Bilotkach, V., 2019. Airline partnerships, antitrust immunity, and joint ventures: What we know and what I think we would like to know. Review of Industrial Organization 54, 37-60.

Bilotkach, V. and Hüschelrath, K., 2011. Antitrust immunity for airline alliances. Journal of Competition Law and Economics 7, 335-380.

Bilotkach, V. and Hüschelrath, K., 2012. Airline alliances, antitrust immunity, and market foreclosure. Review of Economics and Statistics 95, 1368-1385.

Bilotkach, V. and Hüschelrath, K., 2013. Antitrust immunity for airline alliances. Journal of Competition Law and Economics 7, 335-380.

Brueckner, J. K., 2001. The economics of international codesharing: An analysis of airline alliances. International Journal of Industrial Organization 19, 1475-1498.

Brueckner, J. K., 2003. International airfares in the age of alliances: The effects of codesharing and antitrust immunity. Review of Economics and Statistics 85, 105-118.

Brueckner, J. K. and Flores-Fillol, R., 2020. Market structure and quality determination for complementary products: Alliances and service quality in the airline industry. International Journal of Industrial Organization 68, 1-20.

Brueckner, J. K., Lee, D. and Singer, E., 2011. Alliances, codesharing, antitrust immunity, and international airfares: Do previous patterns persist? Journal of Competition Law and Economics 7, 573-602.

Brueckner, J. K. and Pels, E., 2005. European airline mergers, alliance consolidation, and consumer welfare. Journal of Air Transport Management 11, 27-41.

Brueckner, J. K. and Proost, S., 2010. Carve-outs under airline antitrust immunity. International Journal of Industrial Organization 26, 657-668. 
Brueckner, J. K. and Whalen, 2000. The price effects of international airline alliances. Journal of Law and Economics 37, 379-415.

Bulow, J. I., Geanakoplos, J. D. and Klemperer, P. D., 1985. Multimarket oligopoly: Strategic substitutes and complements. Journal of Political Economy 93, 488-511.

Caves, D. W., Christensen, L. R. and Tretheway, M. W., 1984. Economies of density versus economies of scale: Why trunk and local service airline costs differ. RAND Journal of Economics 15, 471-489.

Czerny, A. I., 2009. Code-sharing, price discrimination and welfare losses. Journal of Transport Economics and Policy 43, 193-210.

Czerny, A. I., van den Berg, V. A. C., and Verhoef, E. T., 2016. Carrier collaboration with endogenous fleets and load factors when networks are complementary. Transportation Research Part B 94, $285-297$.

Dixit, A., 1979. A model of duopoly suggesting a theory of entry barriers. The Bell Journal of Economics $10,20-32$.

Douglas, G. W. , Miller, J. C. , 1974. Quality competition, industry equilibrium, and efficiency in the price-constrained airline market. American Economic Review 64, 657-669 .

Gaggero, A. and Bartolini, 2012. The determinants of airline alliances. Journal of Transport Economics and Policy 46, 399-414.

Gayle, P., 2008. An empirical analysis of the competitive effects of the delta/continental/ northwest code-share alliance. Journal of Law and Economics 51, 743-766.

Ito, H. and Lee, D., 2005. Domestic codesharing practices in the US airline industry. Journal of Air Transport Management 11, 89-97.

Ito, H. and Lee, D., 2007. Domestic code sharing, alliances, and airfares in the U.S. airline industry. Journal of Law and Economics 50, 355-380.

Jiang, C., Wan, Y., and D'Alfonso, T., 2015. Strategic choice of alliance membership under local competition and global networks. Journal of Transport Economics and Policy 49, 316-337.

Levitan, R. E. and Shubik, M., 1971. Noncooperative equilibria and strategy spaces in an oligopolistic market. Differential Games and Related Topics, Amsterdam: North-Holland Publishing Company, 429-447.

Miller, J. C. , 1972. A time of day model for aircraft scheduling. Transportation Science 6, 221-246. 
Oum, T., Park, J. and Zhang, A., 1996. The effects of airline codesharing agreements on firm conduct and international air fares. Journal of Transport Economics and Policy 30, 187-202.

Park, J.-H., 1997. The effect of airline alliances on markets and economic welfare. Transportation Research Part E 33, 181-195.

Park, J.-H., and Zhang, A., 2000. An empirical analysis of global airline alliances: Cases in the North Atlantic markets. Review of Industrial Organization 16, 367-384.

Spengler, J. J., 1950. Vertical integration and antitrust policy. Journal of Political Economy 58, 347352.

Vives, X., 1999. Oligopoly pricing: old ideas and new tools. MIT press.

Wan, X., Zou, L., and Dresner, M., 2009. Assessing the price effects of airline alliances on parallel routes. Transportation Research Part E 45, 627-641.

Whalen, W. T., 2007. A panel data analysis of code sharing, antitrust immunity and open skies treaties in international aviation markets. Review of Industrial Organization 30, 39-61.

Zhang, A., and Zhang, Y., 2006. Rivalry between strategic alliances. International Journal of Industrial Organization 24, 287-301.

Zou, L., Oum, T., and Yu, C., 2011. Assessing the price effects of airline alliances on complementary routes. Transportation Research Part E 47, 315-332.

Zhang, A. and Czerny, A. I., 2012. Airports and airlines economics and policy: An interpretive review of recent research. Economics of Transportation 1, 15-34. 


\section{Appendix}

\section{A Proofs}

\section{A.1 Proof of Lemma 1}

To obtain the critical value of the differentiation-parameter $d^{I}$, substitute $d$ by $d^{I}$ and equalize equilibrium profits in (14) and (19), which yields

$$
\frac{2 a^{2}}{9\left(b+d^{I}\right)}-2 F=\frac{a^{2}}{9 b}-F
$$

Solving the equation yields the critical value of the differentiation-parameter, which is given by

$$
d^{I}=\frac{b\left(a^{2}-9 b F\right)}{a^{2}+9 b F} .
$$

The perfect complementarity of the carrier networks, implies that carriers can never increase their own profit by unilaterally deviating and maintaining two connections given that the other carrier maintains one connection. This is because this would not change revenues but increase maintenance costs. This is true independent of whether $d$ is higher or lower then $d^{I}$. However, if the differentiation parameter is small enough in the sense that $d \leq d^{I}$, then carriers cannot increase their own profit by unilaterally deviating from a situation in which both carriers maintain two connections to a situation in which they maintain only one connection and the other carrier maintains two connections. Therefore, if $d \leq d^{I}$, maintaining two connections is an equilibrium solution for both carriers.

\section{A.2 Proof of Lemma 2}

To obtain the critical value of the differentiation-parameter $d^{A}$, substituting $d$ with $d^{A}$ and equalizing equilibrium profits in (27) to (28) yields

$$
\frac{a^{2}}{4\left(b+d^{A}\right)}-2 F=\frac{a^{2}}{8 b}-F .
$$

Solving the equation yields the critical value of the differentiation-parameter, which is given by

$$
d^{A}=\frac{b\left(a^{2}-8 b F\right)}{a^{2}+8 b F} .
$$

The difference between $d^{A}$ and $d^{I}$ is given by

$$
\begin{aligned}
d^{A}-d^{I} & =\frac{b\left(a^{2}-8 b F\right)}{a^{2}+8 b F}-\frac{b\left(a^{2}-9 b F\right)}{a^{2}+9 b F} \\
& =\frac{2 F a^{2} b^{2}}{72 F^{2} b^{2}+17 F a^{2} b+a^{4}}
\end{aligned}
$$

which is positive. 


\section{A.3 Proof of Lemma 4}

To obtain the critical value of the differentiation-parameter $d^{W}$, substituting $d$ with $d^{W}$ and equalizing first-best welfare in (37) and (38) yields

$$
\frac{a^{2}}{b+d^{W}}-2 F=\frac{a^{2}}{2 b}-F
$$

Solving the equation yields the critical value of the differentiation-parameter, which is given by

$$
d^{W}=\frac{b\left(a^{2}-2 b F\right)}{a^{2}+2 b F} .
$$

The difference between $d^{W}$ and $d^{A}$ is given by

$$
\begin{aligned}
d^{W}-d^{A} & =\frac{b\left(a^{2}-2 b F\right)}{a^{2}+2 b F}-\frac{b\left(a^{2}-8 b F\right)}{a^{2}+8 b F} \\
& =\frac{12 F a^{2} b^{2}}{16 F^{2} b^{2}+10 F a^{2} b+a^{4}}
\end{aligned}
$$

which is positive.

\section{A.4 Proof of Lemma 5}

To obtain the critical value of the differentiation-parameter $d^{I W}$, substituting $d$ with $d^{I W}$ and equalizing equilibrium welfares in (27) to (28) yields

$$
\frac{5 a^{2}}{9\left(b+d^{I W}\right)}-2 F=\frac{5 a^{2}}{18 b}-F .
$$

Solving the equation yields the critical value of the differentiation-parameter, which is given by

$$
d^{I W}=\frac{b\left(5 a^{2}-18 b F\right)}{5 a^{2}+18 b F} .
$$

The difference between $d^{I W}$ and $d^{A}$ is given by

$$
\begin{aligned}
d^{I W}-d^{A} & =\frac{b\left(5 a^{2}-18 b F\right)}{5 a^{2}+18 b F}-\frac{b\left(a^{2}-8 b F\right)}{a^{2}+8 b F} \\
& =\frac{44 F a^{2} b^{2}}{144 F^{2} b^{2}+58 F a^{2} b+5 a^{4}}
\end{aligned}
$$

which is positive. The difference between $d^{W}$ and $d^{I W}$ is given by

$$
\begin{aligned}
d^{W}-d^{I W} & =\frac{b\left(a^{2}-2 b F\right)}{a^{2}+2 b F}-\frac{b\left(5 a^{2}-18 b F\right)}{5 a^{2}+18 b F} \\
& =\frac{16 F a^{2} b^{2}}{36 F^{2} b^{2}+28 F a^{2} b+5 a^{4}}
\end{aligned}
$$

which is positive. 


\section{A.5 Proof of Lemma 6}

To obtain the critical value of the differentiation-parameter $d^{A W}$, substituting $d$ with $d^{A W}$ and equalizing welfares in (43) and (44) yields

$$
\frac{3 a^{2}}{4\left(b+d^{A W}\right)}-2 F=\frac{3 a^{2}}{8 b}-F .
$$

Solving the equation yields the critical value of the differentiation-parameter, which is given by

$$
d^{A W}=\frac{b\left(3 a^{2}-8 b F\right)}{3 a^{2}+8 b F} .
$$

The difference between $d^{A W}$ and $d^{I W}$ is given by

$$
\begin{aligned}
d^{A W}-d^{I W} & =\frac{b\left(3 a^{2}-8 b F\right)}{3 a^{2}+8 b F}-\frac{b\left(5 a^{2}-18 b F\right)}{5 a^{2}+18 b F} \\
& =\frac{28 F a^{2} b^{2}}{144 F^{2} b^{2}+94 F a^{2} b+15 a^{4}}
\end{aligned}
$$

which is positive. The difference between $d^{W}$ and $d^{A W}$ is given by

$$
\begin{aligned}
d^{W}-d^{A W} & =\frac{b\left(a^{2}-2 b F\right)}{a^{2}+2 b F}-\frac{b\left(3 a^{2}-8 b F\right)}{3 a^{2}+8 b F} \\
& =\frac{4 F a^{2} b^{2}}{16 F^{2} b^{2}+14 F a^{2} b+3 a^{4}}
\end{aligned}
$$

which is positive. 